\title{
3D Data Analysis for Teeth Morphology Study *
}

\author{
Armen Gaboutchian ${ }^{1}$, Vladimir Knyaz ${ }^{2,3}[0000-0002-4466-244 X]$, Sergey Vasilyev ${ }^{4}$, \\ and Anatoly Maksimov ${ }^{3}$ \\ 1 Peoples' Friendship University of Russia, Moscow, Russia \\ armengaboutchian@mail.ru \\ 2 Moscow Institute of Physics and Technology (MIPT), Dolgoprudny, Russia \\ ${ }^{3}$ State Research Institute of Aviation System (GosNIIAS), 125319 Moscow, Russia \\ $\{$ knyaz, aa.maksimov\}@gosniias.ru \\ http: //www.mipt.ru \\ ${ }^{4}$ Institute of Ethnology and Anthropology of Russian Academy of Sciences \\ vasborleyandex.ru
}

\begin{abstract}
Morphological studies are carried out predominantly in dental or anthropological research not just because these disciplines are directly related to teeth: dental structures are able to provide a wide-range of important information. Traditional odontological (related to teeth) study techniques can be associated, in general, to descriptive and measuring approaches. Descriptive techniques have been always more developed and compensating scarceness of odontometrics, as traditional manual measurements provide definitely insufficient information for morphological assessments - only approximate size evaluations are possible. However, the presented in the article new non-contact odontometric technique has proved its capability to overcome barriers and to improve dental measuring research through obtaining dense and accurate data of significant diversity and amount to turn quantitative assessments of dental structures into qualitative and related to morphology. The method itself is based on improved interpretation of dental morphology providing two basic stages: orientation of 3D models of teeth and measurements on series of its sections. At the final stage odontometric data is captured, re-calculated and subjected to analyses.
\end{abstract}

Keywords: 3D Reconstruction, 3D Surface Analysis, 3D Prototyping, Odontology, 3D Visualization, Automated Digital Odontometry.

\section{Introduction}

The problems of traditional manual odontometry, conducted by calipers, are multiple in its backgrounds as the method, which is still being applied in various disciplines: physical anthropology, forensic dentistry, clinical dentistry or palaeoanthropology [1,2,3],

Copyright (C) 2020 for this paper by its authors. Use permitted under Creative Commons License Attribution 4.0 International (CC BY 4.0).

* The reported study was funded by Russian Foundation for Basic Research (RFBR) according to the research project 17-29-04509. 


\section{A. Gaboutchian et al.}

rises to late XIX century. Some essential modifications refer to 1960s and other suggestions relate to later years [4]. However, this method has been always linked to expertbased estimates of predominantly two parameters, obtained in two, usually and approximately perpendicular dimensions; the measurement accuracy has been accepted as 0.1 $\mathrm{mm}$. The described features turn traditional odontometry into knowledge of a limited number of experts as tooth orientating and measuring requires special skill, nevertheless it still possesses certain degree of subjectivity and difficulties in verifying results. At the same time two, or even sometimes several, parameters are insufficient for comprehensive morphological description of the studied tooth. Thus only general assessments are possible: the tooth is small or large; or the tooth is wide rather than long, etc. Under such circumstances descriptive techniques come to the fore in odontological studies as there are much more morphological features which can be described for teeth in terms of their expression degree (however, again, expertly assessed) than measured.

Nevertheless there was need for objective, accurate and diverse range of based on measurements data in odontological studies, which had become an encouraging factor for elaborating of the presented method. The methodological framework of the presented technique was initially developed for studies of tooth preparation procedure in dentistry [5,6]; and now its applications include dental, archaeological and palaeoanthropological research, providing odontometric data corresponding to observed dental morphological features $[7,8,9]$. Today the process of measurements is fully automated and consists of two stages: orientation and measurements per se; both are based on the suggested and successfully tested interpretations of dental morphology. The method of automated digital odontometry can be applied only to 3D reconstructions of tooth surface and hence depends on the method and accuracy of imaging and image processing. Subsequently 3D images of teeth are uploaded and subjected to surface analysis in order to obtain correct of the studied object. The orientation algorithms set correct directions of sectioning planes crossing teeth in appropriate direction for obtaining a series of contours, which, in their turn, provide for study objectivity and consistency. The contours serve for obtaining odontometric data by means of geometric constructions. At the next stage the parameters are used for calculations and analyses.

\section{Related work}

Measurements are at the core of numerous odontological studies in many disciplines. Traditionally carried out by calipers [10], they increasingly tend to methods, based on $3 \mathrm{D}$ imaging, image processing and surface analysis, which is evaluated positively in comparative studies [11]. At a minimum it is possible to use general advantages of digital methods by application of traditional techniques to 3D reconstructions of tooth surfaces [12]. However more profound surface analysis methods have been used as well in odontological research $[13,14,15,16]$.

There is a variety of image obtaining techniques applicable for odontological studies, as well as for automated digital odontometry presented in the paper. Optical methods of scanning are represented predominantly by devices used in dental practice. In case of plaster model scanning, dental laboratory scanners using different types of lighting are found to be the most relevant. With intraoral scanners are applied for natural - 
covered with partially translucent enamel - teeth imaging [17,18]. On the other had $\mathrm{x}$-ray based techniques, especially micro-CT scanners, provide accurate and essential data on outer and inner structures of teeth [19]. There are examples of contact scanning by piezoelectric digitizer application in odontological studies as well [20]. Taking into consideration a wide variety of imaging methods studies of accuracy are essential in odontological research based on objectivity of assessments [21].

Reconstructed surfaces of teeth are quite often studied as whole; however sectioning is wide-spread approach as well. And among sections those which are oriented in vestibular-oral direction are the most informative due to dental morphological framework. Thus sectioning (performed, however, in a "manual" mode, in contrast to the presented in this article method) is used, as an example, in enamel thickness studies [22] or studies referred to dental wear [23]. Measure-ment automation and appropriately functioning algorithms starts playing an essential role in study objectivity if compared to expert-based research, when discrepancies accompany different attempts to measurements, as well as results vary between experts [24].

Application of 3D imaging and image processing techniques allows measurements beyond traditional odontometric parameters: maximal tooth crown dimensions in mesiodistal and bucco-lingual directions. And here angular measurements play a significant role due inclinations of dental cusp planes, including studies of dental wear is [25]. Regarding dental wear (or usually natural functional attrition of opposing and adjacent teeth) should be mentioned that it can change dental morphology substantially, thus 3D imaging and processing methods provide for studies of inner morphological structures located at the enamel-dentine junction [26,27].

\section{Methods and material}

As we have mentioned automated digital odontometry has been applied in studies referring to different material such as plaster models of dental arches (including mounting them in closure positions), separate teeth, teeth with bone structures and others. And therefore different imaging techniques have been implemented, corresponding to scanned objects and study objectives. Initially 3D imaging was performed on photogrammetric system designed at GosNIIAS [28,6]; however plaster models and some natural structures (when it was possible to cover them permanently with opaque spray coating to exclude enamel translucency) were scanned on dental laboratory scanners providing high accuracy (S600 Arti Scanner; Zfx Evolution plus); however we should take into consideration that plaster model fabrication process inevitably causes certain distortions due to shrinkage of the used materials. Scanning of unique palaeoanthropological findings required application of intraoral scanners, providing high accuracy on limited scanning area. As an example Trios, 3Shape intraoral scanner was used; its construction includes combination of specific lighting and confocal optics. Though it is difficult to clearly estimate its scanning accuracy as it depends on many factors, including optical features of the object, scanning area (preferably within 3-5 units) or operator skills, surface reconstruction details appear to be sufficient for the proposed method application. It should be mentioned that X-ray based methods have been used for imaging as well, which provides the opportunity for comparisons with optical reconstructions, 


\section{A. Gaboutchian et al.}

due to defined and regular slice thickness. As for cone-beam computed tomography, which has performed effectively in a number of archaeological and anthropological studies, even at its highest resolution (up to $80 \mu \mathrm{m}$ ) it possesses insufficient surface reconstruction accuracy if compared to optical scans (Figure 1(a)). Moreover, application of standard approaches to surface smoothing eliminates essential morphological features of the studied teeth. Therefore automated digital odontometry required micro-CT scans in order to reach the necessary degree of accuracy and detailed reconstruction of tooth surface. Micro-CT scans have been applied to separate teeth (Skyscan 1174); and the latest advances refer to obtaining complete dental arches scans with skulls related to Upper Palaeolithic individuals from Sunghir' (GE v|tome $\mid \mathrm{x} \mathrm{m}$ ). And regarding accuracy of the above-mentioned micro-CT scans, which again depends on object volume, it is within limits of 10 and $45 \mu \mathrm{m}$; optical reconstructions can be compared in details with the last.

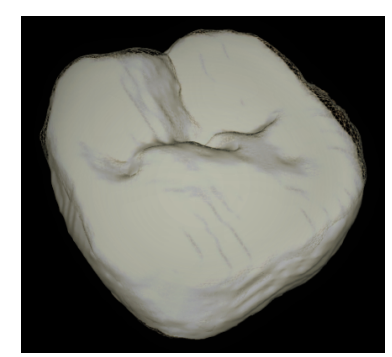

(a)

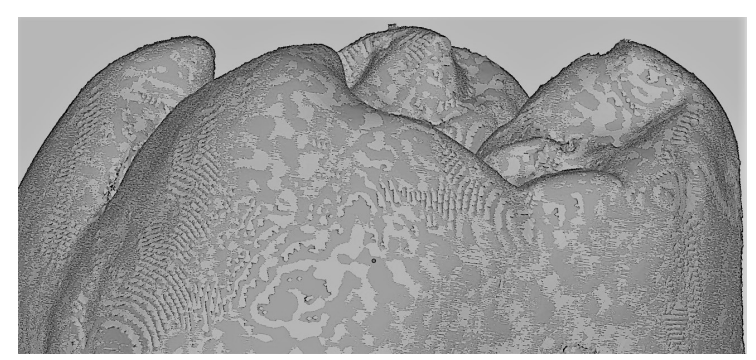

(b)

Fig. 1. (a) Segmented and combined CBCT and optical scans of Early Bronze molar, (b) Global smoothing of enamel surface on micro-CT scan.

The obtained scans required image processing including volume rendering, segmentation of teeth, surface smoothing, changes of format and simple trimming (Figure $1(\mathrm{~b})$ ).

At the initial stage of automated digital odontometry tooth orientation is performed (Algorithm 1). The algorithm is automated and based on 3D surface analysis including calculation of curvature coefficient and subsequent demarcation of structure borders on the studied tooth. Tooth orientation provides for setting directions of axes responsible for executing the following stage of odontotomy - "slicing" teeth by equally spaced parallel planes [29]. Measurements on tooth contours obtained through sectioning are performed subsequently in an automated mode. The obtained parameters are re-calculated in order to increase the study range, which can be presented as linear (in several directions), contour, angular, area and other parameters $\mathbf{P}_{o}=\left\{p_{j}\right\}$, which are preserved for further analysis. In some cases automated process is interrupted for control reasons and necessary "manual" adjustments. 


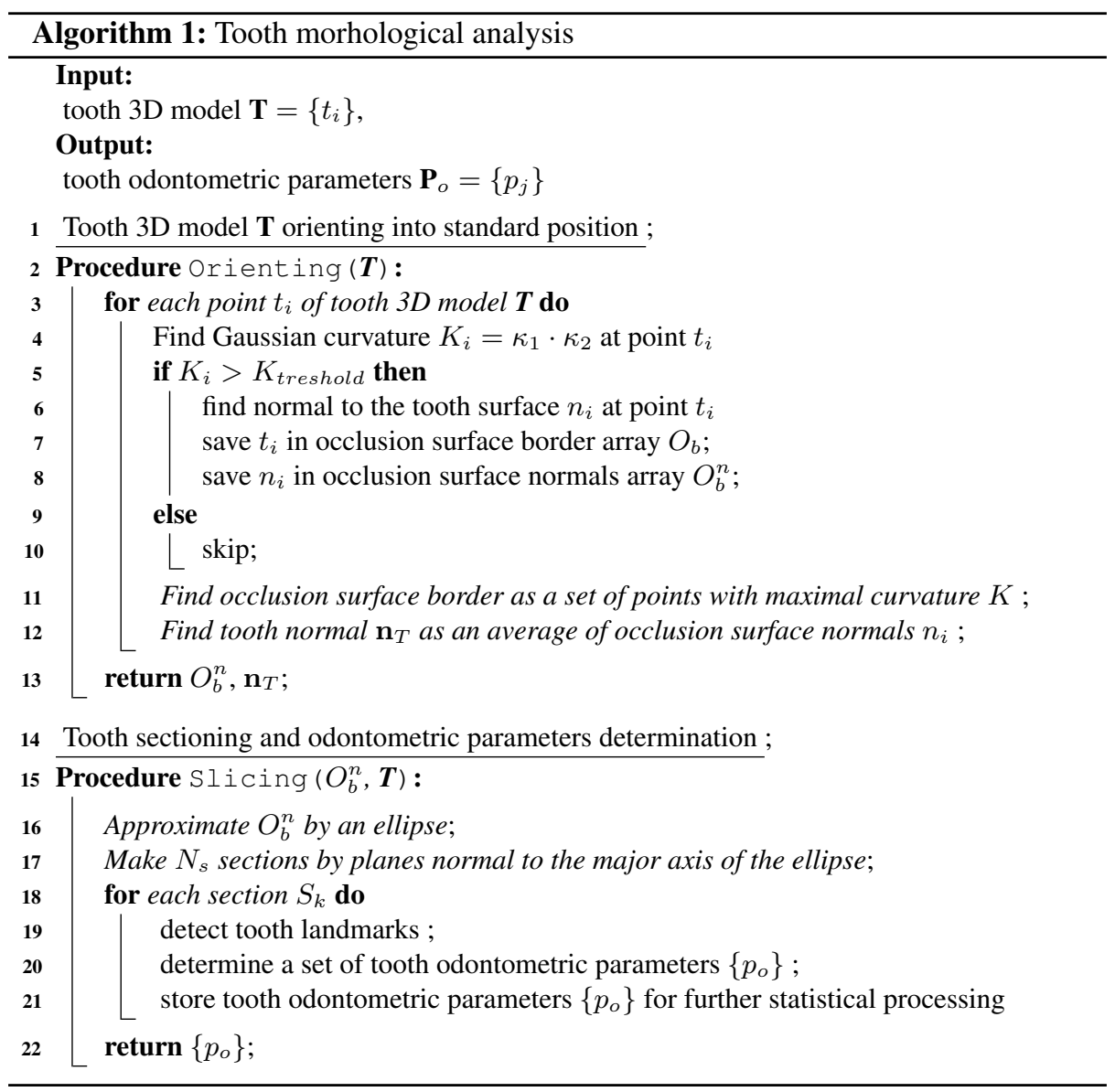

\section{Results}

Morphological features of teeth already described by non-metric methods form a very wide variety and represent a complex relief; at the same time they are essential in terms of biological interpretations, which are however subjective to some extent. Nevertheless the suggested method of automated digital odontometry provides for obtaining objective measurements even in rather complicated circumstances and in addition possesses high sensitivity. This can be shown on two examples: a tooth from Bronze Age Shengavit (Table 1), and the above-mentioned Upper Palaeolithic samples which have a feature described as sharpened cusp tips with inner, or central, inclination.

Samples from Shengavit were measured manually and furthermore scanned by two different intraoral scanners; each of the scans served for automated digital measurements. The results demonstrate more visible discrepancies between manual and digital measurements than in between two digital. However similar algorithms still differ, however not by much in terms of results, on two different surface reconstructions. Regarding 
6 A. Gaboutchian et al.

Table 1. Measurements of vestibular-lingual tooth crown diameter; Shengavit, M2d.

\begin{tabular}{lr}
\hline \hline Parameter & Value \\
\hline Automated Digital Measurements & Tooth $4.7\left(M_{2} d\right)$ \\
\hline VL max, mm; (Trios, 3Shape) & 9.86 \\
VL max, mm; (Omnicam, CEREC) & 9.85 \\
\hline Manual Measurements & Tooth $\left.4.7 M_{2} d\right)$ \\
\hline VL max, mm & 9.7 \\
\hline \hline
\end{tabular}

Sunghirian findings, both second molars of the studied individual possess unusual morphology, however unevenly expressed on different cusps of right and left tooth (Figure 2).

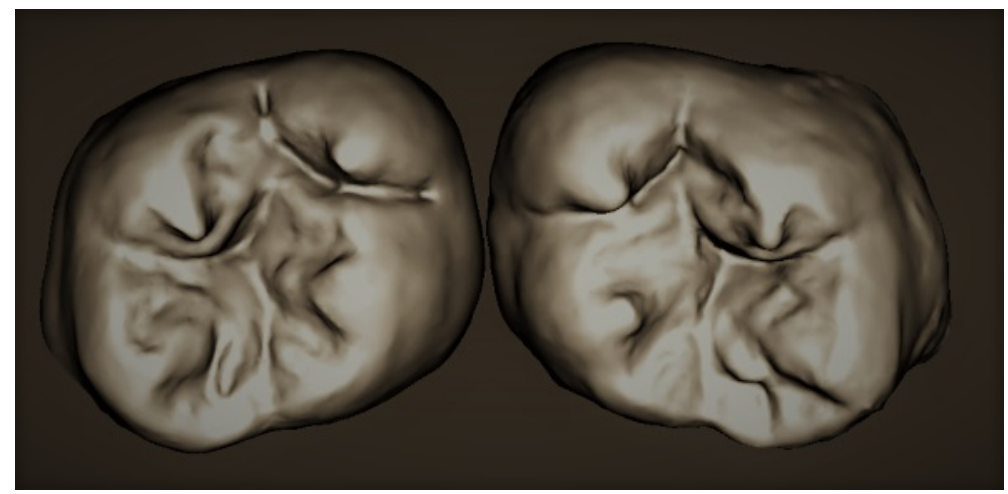

Fig. 2. Right and left upper molars with sharp bent cusps.

The suggested algorithms perform automated orientation of the teeth and based on successive stages of 3D surface analysis (Figure 3).

Tooth sectioning provides contour obtaining and following measurements; thus a wide range of parameters were obtained for further morphological comparisons (Figure 4). Parameters obtained on sections serve for calculation of average parameters referring to parts of teeth or teeth as a whole.

Without expanding too much on odontometric data in the current paper we should mention that the proposed measurement results correspond to the observed morphological features. Moreover, various parameters - average, referring to teeth, their cusps, cusp slopes and anatomical occlusal surfaces: linear in vertical and horizontal direction, angular and contour, provide sufficient data to depict differences between two antimere teeth, both possessing the rare morphological feature, though different in expression degree and location (Table 2). 


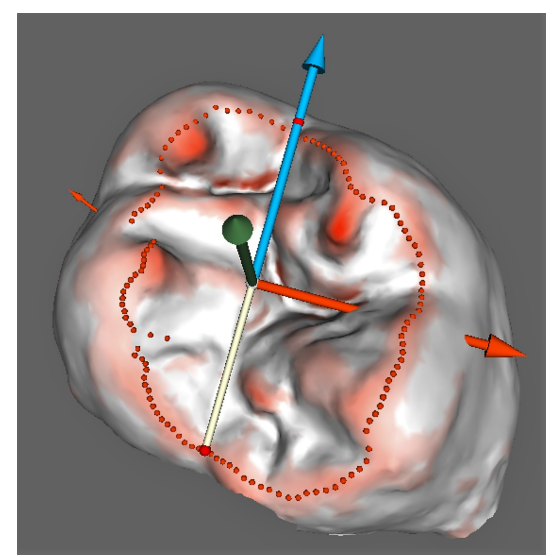

Fig. 3. Orientation of the measured molar.

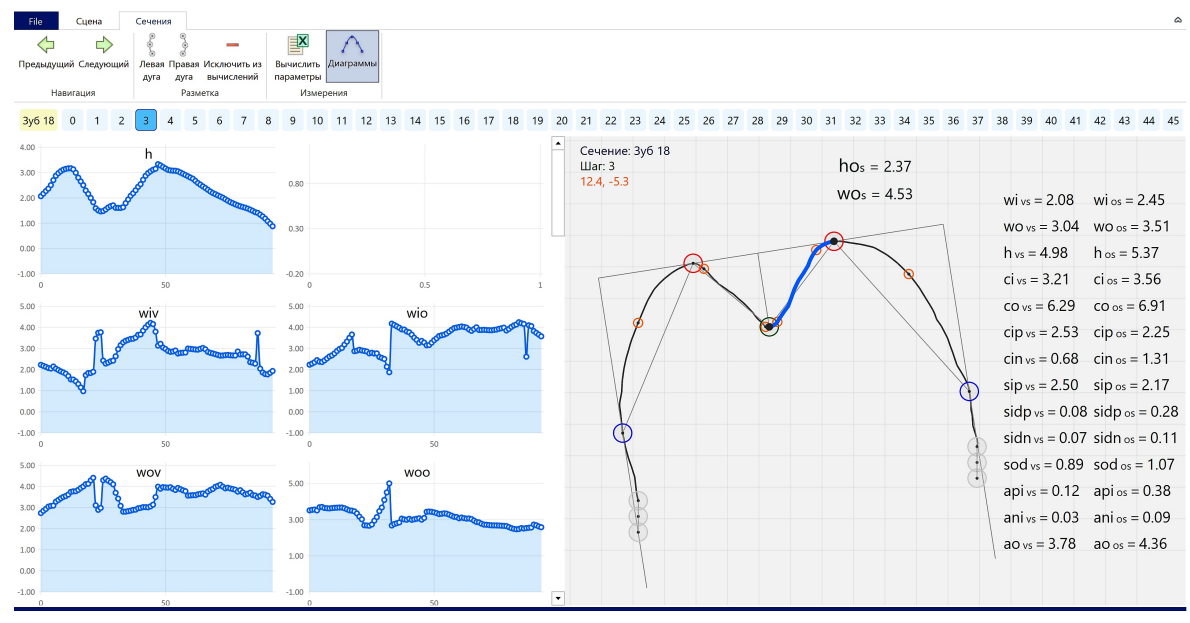

Fig. 4. Software interface with contour measurements and parameters.

\section{Conclusion}

The developed non-contact odontometric technique for tooth morphology study provides new possibilities which are unavailable in manual measurements. This technique improves dental measuring research through obtaining dense and accurate data of significant diversity and amount to turn quantitative assessments of dental structures into qualitative and related to morphology. The method itself is based on improved interpretation of dental morphology providing two basic stages: orientation of 3D models of teeth and measurements on series of its sections. The odontometric data captured in such way is post processed for analysis. 
8 A. Gaboutchian et al.

Table 2. Odontometric data: absolute average values

\begin{tabular}{|c|c|c|}
\hline \multirow[t]{2}{*}{ Parameter } & \multicolumn{2}{|l|}{ Value } \\
\hline & \multicolumn{2}{|c|}{ right tooth left tooth } \\
\hline \multicolumn{3}{|c|}{ linear horizontal, mm } \\
\hline tooth & 12,71 & 12,34 \\
\hline oral cusps (oc) & 6,57 & 6,14 \\
\hline oc oral slopes & 3,95 & 3,39 \\
\hline oc vest.slopes & 2,62 & 2,75 \\
\hline vest. cusps (vc) & 6,14 & 6,20 \\
\hline vc oral slopes & 3,33 & 3,04 \\
\hline vc vest.slopes & 2,80 & 3,16 \\
\hline occl. surface & 5,95 & 5,79 \\
\hline \multicolumn{3}{|c|}{ linear vertical, $\mathrm{mm}$} \\
\hline occl. surface & 2,25 & 2,13 \\
\hline \multicolumn{3}{|c|}{ contour length, $\mathrm{mm}$} \\
\hline tooth & 22,70 & 21,67 \\
\hline oral cusps (oc) & 11,51 & 10,12 \\
\hline oc oral slopes & 7,83 & 6,47 \\
\hline oc vest.slopes & 3,69 & 3,65 \\
\hline vest. cusps (vc) & 11,19 & 11,54 \\
\hline vc oral slopes & 4,30 & 4,05 \\
\hline vc vest.slopes & 6,89 & 7,49 \\
\hline occl. surface & 7,99 & 7,71 \\
\hline \multicolumn{3}{|l|}{ angular, deg } \\
\hline oral cusps (oc) & 82,8 & 86,2 \\
\hline oc oral slopes & 33,0 & 34,2 \\
\hline oc vest.slopes & 49,8 & 52,0 \\
\hline vest. cusps (vc) & 82,2 & 81,6 \\
\hline vc oral slopes & 56,2 & 54,8 \\
\hline vc vest.slopes & 26,0 & 26,8 \\
\hline occl. surface & 106,0 & 106,8 \\
\hline
\end{tabular}

\section{Acknowledgements}

The reported study was funded by Russian Foundation for Basic Research (RFBR) according to the research project 17-29-04509.

\section{References}

1. Benazzi, S., Coquerelle, M., Fiorenza, L., Bookstein, F., Katina, S., Kullmer, O. Comparison of Dental Measurement Systems for Taxonomic Assignment of First Molars. American journal of physical anthropology. 144. 342-54. 10.1002/ajpa.21409

2. Khangura, R.K., Sircar, K., Singh, S., Rastogi, V. Sex determination using mesiodistal dimension of permanent maxillary incisors and canines. Journal of forensic dental sciences. 3 . 81-5. 10.4103/0975-1475.92152, 2011 
3. Song, J.-W., Leesungbok, R., Park, S.-J., Chang, S., Ahn, S.-J., Lee, S.-W. Analysis of crown size and morphology, and gingival shape in the maxillary anterior dentition in Korean young adults. The Journal of Advanced Prosthodontics. 9. 315. 10.4047/jap.2017.9.4.315, 2017

4. Irish J.D., Scott G.R. A companion to dental anthropology. John Wiley \&Sons, 2016, Online ISBN: 9781118845486. doi:10.1002/9781118845486, 2015

5. Knyaz V., Zheltov S., Gabuchyan A., Bolshakov G. Photogrammetric system for automated teeth arches 3D models generation and teeth occlusion analysis. Op-tical 3D Measurement Techniques VIII, Zurich, 2007, Vol. I, pp. 299-304.

6. Knyaz, V. A.: Image-based 3D reconstruction and analysis for orthodontia, Int. Arch. Photogramm. Remote Sens. Spatial Inf. Sci., XXXIX-B3, 585589, https://doi.org/10.5194/isprsarchives- XXXIX-B3-585-2012, 2012.

7. Gaboutchian, A. V., Knyaz, V. A., Leybova, N. A., Petrosyan, G., Simonyan, H., and Vasilyev, S. V.: Application of photogrammetric techniques in pal-aeoodontological studies through automated digital shape analysis of human teeth. Int. Arch. Photogramm. Remote Sens. Spatial Inf. Sci., XLII-2/W12, 75-80, https://doi.org/10.5194/isprs-archives-XLII-2W12-75-2019

8. Gaboutchian, A. V., Knyaz, V. A., Apresyan S. Automated Digital Odontometric Study Of Manual And Computer-aided Methods Of Tooth Crown Modelling In Dentistry. The International Archives of the Photogrammetry, Remote Sensing and Spatial Information Sciences, Volume XLII-2/W12, 2019 Int. Worksh. on "Photogrammetric \& Computer Vision Techniques for Video Surveillance, Biometrics and Bio-medicine", 13-15 May 2019, Moscow, Russia, https://doi.org/10.5194/isprs-archives-XLII-2-W12-69-2019

9. Gaboutchian, A. V., Knyaz, V. A., Novikov, M. M., Vasilyev, S. V., Leybova, N. A., Korost, D. V., Cherebylo, S. A., and Kudaev, A. A.: AUTOMATED DIGITAL ODONTOMETRY: MEASUREMENT DATA ANALYSES IN CASES OF COMPLICATED DENTAL MORPHOLOGY, Int. Arch. Photogramm. Remote Sens. Spatial Inf. Sci., XLIII-B2-2020, 851-856, https://doi.org/10.5194/isprs-archives-XLIII-B2-2020-851-2020, 2020.

10. Dash, K., Panda, A., Behura, Sh., Ramachandra, S., Bhuyan, L., Ban-dopadhyay, A. Employing dimensional disparity of teeth to establish the gender in Odisha population: A dimorphic study. Journal of International Society of Preventive and Com-munity Dentistry. 8. 174-178. 10.4103/jispcd.JISPCD-42-18, 2018

11. Naidu, D., Freer, T. The evidence supporting methods of tooth width measurement: Part I. Vernier calipers to stereophotogrammetry. Australian orthodontic journal. 29. 159-63, 2013

12. Sassani, S., Patel, D., Farella, M., Henneberg, M., Ranjitkar, S., Yong, R., Swindells, S., Brook, A. (2018). Variation in tooth crown size and shape are outcomes of the complex adaptive system associated with the tooth number variation of hypodontia. International Journal of Design \& Nature and Ecodynamics. 13. 114-120. 10.2495/DNE-V13-N1-114-120

13. Guy F., Gouvard F., Boistel R., Euriat A., Lazzari V.. Prospective in (Primate) Dental Analysis through Tooth 3D Topographical Quantification. PloS one, 2013. 8. e66142. 10.1371/journal.pone.0066142., 2013.

14. Braga, J., Zimmer, V., Dumoncel, J., Samir, Ch., de Beer, F., Zanolli, C., Pinto, D., Rohlf, F., Grine, F. Efficacy of diffeomorphic surface matching and 3D geometric morphometrics for taxonomic discrimination of Early Pleistocene hominin mandibular molars. Journal of Human Evolution. 130. 21-35. 10.1016/j.jhevol.2019.01.009. 2019.

15. Choi, G., Chan, H.L., Yong, R., Ranjitkar, S., Brook, A., Townsend, G., Chen, K., Lui, L.M. Tooth morphometry using quasi-conformal theory. arXiv:1901.01651v1 [cs.CV], 2019.

16. Dykes and Pilbrow, 2019. Dykes, S. and Pilbrow, V. A mathematical landmark-based method for measuring worn molars in hominoid systematics. PeerJ Vol. 7: e6990 http://doi.org/10.7717/peerj.6990, 2019. 
10 A. Gaboutchian et al.

17. Richert, R. Goujat, A. Venet, L. Viguie, G. Viennot, S. Robinson, P. Farges, J.-Ch. Fages, M., Ducret, M. Intraoral Scanner Technologies: A Review to Make a Successful Impression. Journal of Healthcare Engineering. 2017. 1-9. 10.1155/2017/8427595, 2017.

18. Abduo, J., Elseyoufi, M. Accuracy of Intraoral Scanners: A Systematic Review of Influencing Factors. The European journal of prosthodontics and restorative dentistry. 26. 10.1922/EJPRD_01752Abduo21, 2018.

19. Maret, D., Peters, O., Galibourg, A., Dumoncel, J., Esclassan, R., Kahn, J.-L., Sixou, M., No, T. Comparison of the Accuracy of 3-dimensional Cone-Beam Com-puted Tomography and Micro-Computed Tomography Reconstructions by Using Different Voxel Sizes. Journal of Endodontics. 40. 10.1016/j.joen.2014.04.014, 2014.

20. Benazzi, S., Fantini, M., De Crescenzio, F., Persiani, F., Gruppioni, G. Improving the spatial orientation of human teeth using a virtual 3D approach. Journal of human evolution. 56. 286-93. 10.1016/j.jhevol.2008.07.006.

21. Jacquet, W., Nyssen, E., Ibel, G. Vande Vannet, B. (2013). On the augmented reproducibility in measurements on 3D orthodontic digital dental models and the definition of feature points. Aust Orthod J. 29. 28-33, 2013.

22. Smith, T., Olejniczak, A., Zermeno, J., Tafforeau, P., Skinner, M., Hoffmann, A., Radovčić, J., Toussaint, M., Kruszynski, R., Menter, C., Moggi-Cecchi, J., Glasmacher, U., Kullmer, O., Schrenk, F., Stringer, Ch., Hublin, J-J. Variation in enamel thickness within the genus Homo. Journal of human evolution. 62. 395-411. 10.1016/j.jhevol.2011.12.004, 2012.

23. Oxilia, G., Bortolini, E., Martini, S., Papini, A., Boggioni, M., Buti, L., Figus, C., Sorrentino, R., Townsend, G., Kaidonis, J., Fiorenza, L., Cristiani, E., Kullmer, O., Moggi-Cecchi, J., Benazzi, S. (2017). The physiological linkage between molar incli-nation and dental macrowear pattern. American Journal of Physical Anthropology. 166. 10.1002/ajpa.23476, 2017.

24. Bailey, Sh., Pilbrow, V., Wood, B. Interobserver error involved in in-dependent attempts to measure cusp base areas of Pan M1s. Journal of anatomy. 205. 323-31. 10.1111/j.00218782.2004.00334.x., 2004.

25. Knight-Sadler J., Fiorenza L., Tooth Wear Inclination in Great Ape Molars. Folia Primatologica, 2017. 88. 223-236. 10.1159/000478775.

26. Ortiz A., Skinner M., Bailey Sh., Hublin JJ., Carabelli's trait revisited: An examination of mesiolingual features at the enamel-dentine junction and enamel surface of Pan and Homo sapiens upper molars. Journal of human evolution, 2012. 63. 586-96. 10.1016/j.jhevol.2012.06.003, 2012.

27. Martin, R.,Hublin, J., Gunz, P., Skinner, M. (2017). The morphology of the enamel-dentine junction in Neanderthal molars: Gross morphology, non-metric traits, and temporal trends. Journal of Human Evolution. 103. 20-44. 10.1016/j.jhevol.2016.12.004.

28. Knyaz, V.A., Zheltov, S.Yu.: Photogrammetric techniques for dentistry analysis, planning and visualisation. International Archives of the Photogrammetry, Remote Sensing and Spatial Information Sciences - ISPRS Archives Volume 37, 2008, Pages 783-788 2008 21st ISPRS International Congress for Photogrammetry and Remote Sensing; Beijing; China; 3 July 2008 - 11 July 2008 ;

29. Knyaz, V. A. and Gaboutchian, A. V.: PHOTOGRAMMETRY-BASED AUTOMATED MEASUREMENTS FOR TOOTH SHAPE AND OCCLUSION ANALYSIS, Int. Arch. Photogramm. Remote Sens. Spatial Inf. Sci., XLI-B5, 849-855, https://doi.org/10.5194/isprs-archives-XLI-B5-849-2016, 2016. 